\title{
Energy Spectrum and Mass Composition of Ultra-High Energy Cosmic Rays Measured by the hybrid technique in Telescope Array
}

\author{
Daisuke Ikeda $^{* a}$ and William Hanlon ${ }^{b}$ for the Telescope Array Collaboration ${ }^{\dagger}$ \\ ${ }^{a}$ Institute for the Cosmic Ray Research, University of Tokyo, Chiba, Japan \\ ${ }^{b}$ High Energy Astrophysics Institute and Department of Physics and Astronomy, University of \\ Utah, Salt Lake City, Utah, USA \\ E-mail: ikeda@icrr.u-tokyo.ac.jp
}

\begin{abstract}
The energy spectrum and mass composition of Ultra-High Energy Cosmic Rays (UHECRs) above $10^{18.2} \mathrm{eV}$ from the Telescope Array (TA) utilizing 6 years of hybrid data are presented. TA is a hybrid experiment consisting of fluorescence detectors (FDs) and surface detectors (SDs). We have measured the energy spectrum and mass composition using a hybrid analysis technique which uses information from both FDs and SDs. The obtained energy spectrum with a $21 \%$ energy scale uncertainty is in agreement with the results of the HiRes experiment. The mass composition measurement performed by observing the depth of shower maximum $\left(\mathrm{X}_{\max }\right)$ is consistent with a light primary model.
\end{abstract}

The 34th International Cosmic Ray Conference,

30 July- 6 August, 2015

The Hague, The Netherlands

\footnotetext{
*Speaker.

${ }^{\dagger}$ For full author list and acknowledgments see http://www.telescopearray.org/images/papers/ICRC2015-authorlist.pdf
} 


\section{Introduction}

The Telescope Array (TA) is designed to explore the origin of ultra-high energy cosmic rays (UHECRs) and the mechanisms of production, acceleration at the sources, and propagation in the inter-galactic space. The experiment operates as a "hybrid" observation with the fluorescence detector (FD) and surface detector (SD). The SD consists of two layers of $3 \mathrm{~m}^{2}$ plastic scintillators to detect the secondly particles on the ground induced by the UHECR air showers. The 507 SDs were deployed on a square grid with $1.2 \mathrm{~km}$ spacing, covering an area of $\sim 760 \mathrm{~km}^{2}$. The three FDs are located around the SDs. The FD measures longitudinal development and primary energies of air showers by using the fluorescence photons emitted by atmospheric molecules excited by the secondly particles in the showers. The Black Rock Mesa (BRM) and Long Ridge (LR) stations are located to the southeast and southwest of the TA SD array, respectively.

The energy spectrum and mass composition are important keys to understanding the origin of the UHECR. Using the technique to observe fluorescence photons, we measured the longitudinal development of the air shower directly. In particular, the depth at the maximum shower development, $X_{\max }$, is known as a sensitive parameter for the mass composition of the UHECR. The energy of the UHECR can be measured as a calorimetric way which is less dependence on the high energy interaction model. Therefore, the FD has rolls to determine the energy scale and measure the mass composition in the TA.

In addition, information of the SDs is useful to improve the reconstruction for the FD events. By our Monte-Carlo (MC) study, this hybrid reconstruction significantly improves the accuracy of the energy and $X_{\max }$ from the FD monocular reconstruction.

In this paper, we present the performance of the developed hybrid reconstruction. The measured energy spectrum and mass composition by using the hybrid events of two FDs (BRM and LR) and the SD array in six years of TA operation are also presented.

\section{Hybrid analysis}

We analyse the hybrid data collected at BRM, LR and SD. At first the shower geometry, direction and arrival position on the ground are reconstructed by using pointing direction and arrival timing at the PMTs:

$$
T_{\text {exp }, i}=T_{\text {core }}+\frac{\sin \psi-\sin \alpha_{i}}{c \sin \left(\psi+\alpha_{i}\right)} R_{\text {core }}
$$

where $T_{\exp , i}$ and $\alpha_{i}$ are the expected timing and elevation angle in the SDP for the $i$-th PMT, respectively, $T_{\text {core }}$ is the time when the air shower reached the ground, $R_{\text {core }}$ is the distance from the FD station to the core, and $\psi$ is the elevation angle of the air shower in the SDP (Figure 1). The timing information of the SD near the core is also added to this function.

Second the longitudinal development of the air shower is reconstructed via an inverse MonteCarlo (IMC) technique, which is the comparison of the observed signal in each pixel between data and MC generated by the Gaisser-Hillas function:

$$
N\left(X ; X_{\max }, X_{0}, \Lambda\right)=N_{\max }\left(\frac{X-X_{0}}{X_{\max }-X_{0}}\right)^{\left(X_{\max }-X_{0}\right) / \Lambda} e^{\left(X_{\max }-X\right) / \Lambda},
$$




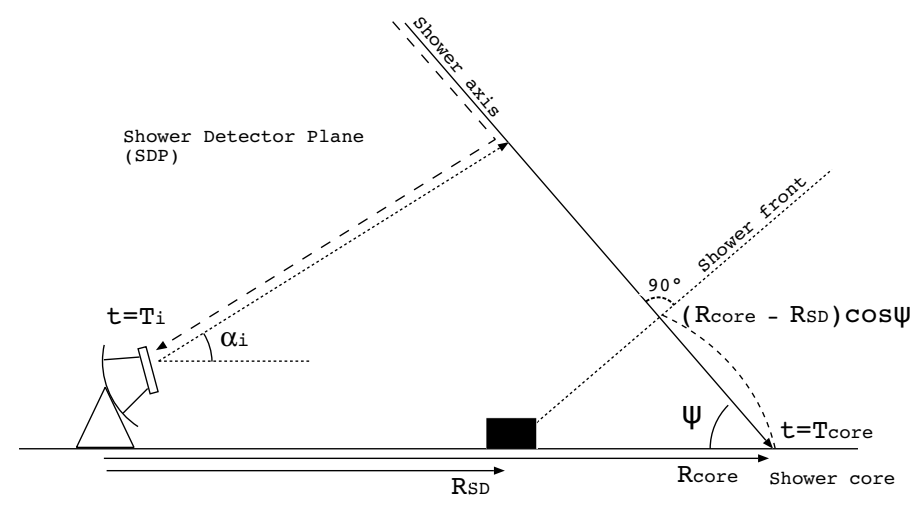

Figure 1: Diagram indicating the Shower Detector Plane (SDP) used in the time fit.

where $X$ is the atmospheric depth, $X_{\max }$ is the depth at the shower maximum, $\Lambda$ is the interaction length of the shower particles, and $X_{0}$ is the offset of $X$. After determination of the $X_{\max }$ by above processes, the energy of the UHECR is obtained by the integration of the Gaisser-Hillas function with the "missing" energy correction which corresponds to the invisible energy deposit mainly coming from the neutrinos. The details of the analysis procedure are described in [1].

To ensure reconstruction quality, we only accept events that satisfy the criteria. For the spectrum analysis, the criteria is follow;(1) the number of PMTs used in the reconstruction is greater than 20, (2) the zenith angle of the reconstructed shower axis is less than 55 degrees, (3) the shower core is inside the edges of the SD array, (4) the angle between the reconstructed shower axis and the telescope is greater than 20 degrees, (5) $X_{\max }$ is observed in the field of view of the telescopes. For the mass composition analysis, we applied additional criteria;(6) the $\chi^{2}$ for the geometry fit is less than 10, (7) the $\chi^{2}$ for the longitudinal profile fit is less than 5, (8) the track length is greater than 10 degrees, (9) the $\psi$ is less than 130 degrees, (10) the time extend of the track is more than 7 $\mu \mathrm{s}$.

In this analysis, we use the fluorescence yield model reported by Kakimoto et al. [2] with the spectrum measured by the FLASH experiment [3]. The atmospheric transparency is measured by the LIDAR system in the TA site [4]. Other applied calibration factors are described in [5]. The systematic error of the energy determination is evaluated to be $21 \%$ [1].

The performance of the reconstruction, the aperture for the energy spectrum and expected $\mathrm{X}_{\max }$ profile are evaluated by using CORSIKA-based air shower simulator [6] with the detector simulation both for SD and FD. Here we used proton and iron primary particles with QGSJET-II03 hadronic interaction model. We checked the quality of the generated MC events by comparing important parameters between data and MC as shown in (Figure 2). By using this MC set, we obtained the resolutions as 0.9 degrees for the arrival direction, $7 \%$ for the energy and $20 \mathrm{~g} / \mathrm{cm}^{2}$ for the $\mathrm{X}_{\max }$.

\section{Energy spectrum}

The use the data set collected for a period of six years from May 112008 to May 112014. The total exposure time with the clear night is about 3400 hours. By the analysis procedure de- 

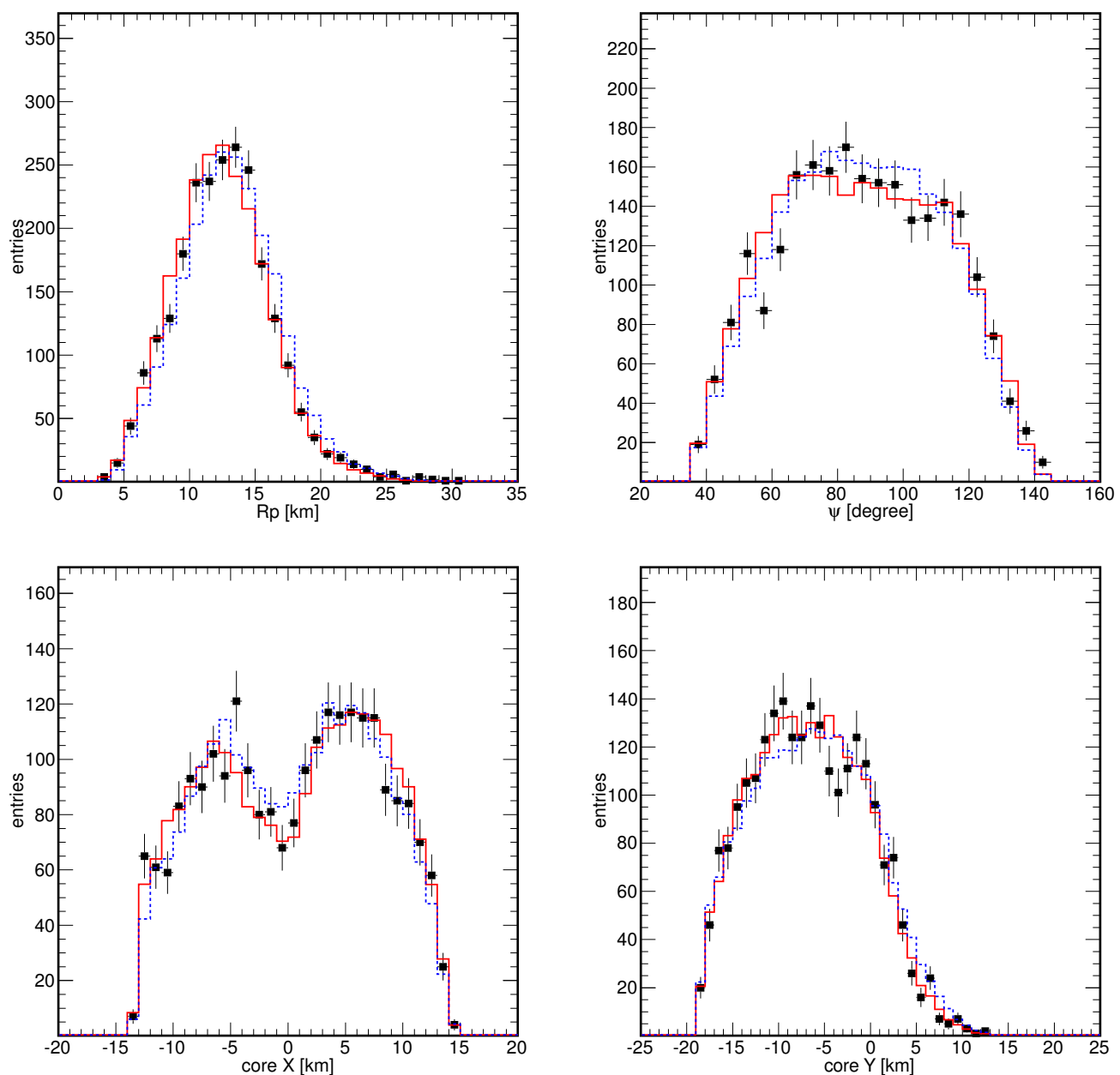

Figure 2: The comparison of the obtained parameters, $\mathrm{Rp}, \psi$, core positions along $\mathrm{X}$ (west to east) and $\mathrm{Y}$ (south to north) between data and MC events above $10^{18.2} \mathrm{eV}$.

scribed above, 2375 events remained above $10^{18.2} \mathrm{eV}$. The exposure obtained from MC events is $\sim$ $1.1 \times 10^{16} \mathrm{~m}^{2} \mathrm{sr} \mathrm{s}$ at $10^{19} \mathrm{eV}$. The obtained energy spectrum is shown in Figure 3. The TA hybrid spectrum are in agreement with the HiRes results.

\section{Mass Composition}

The expected mean $\mathrm{X}_{\max }$ is obtained from the MC events which is analyzed just same procedure as the observed data. The comparison of the observed mean $\mathrm{X}_{\max }$ above $10^{18.2} \mathrm{eV}$ between data and MC is shown in Figure 4.

\section{Summary}

The energy spectrum and $\mathrm{X}_{\max }$ of UHECR above $10^{18.2} \mathrm{eV}$ obtained from six years of TA hybrid data are presented. The obtained energy spectrum is in agreement with the HiRes results 


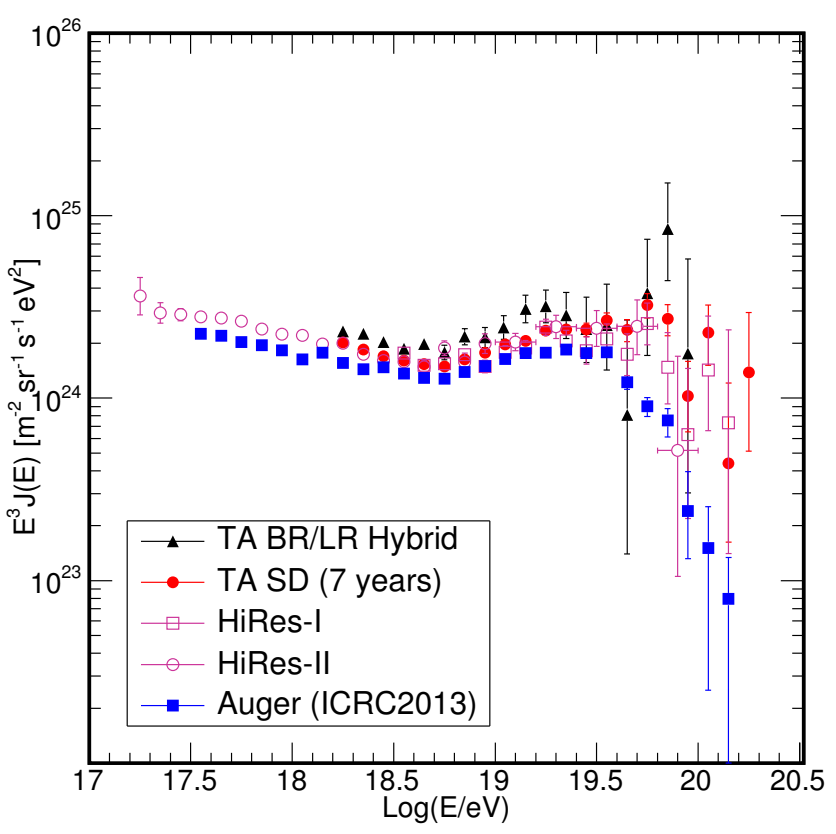

Figure 3: The energy spectra multiplied by $\mathrm{E}^{3}$.

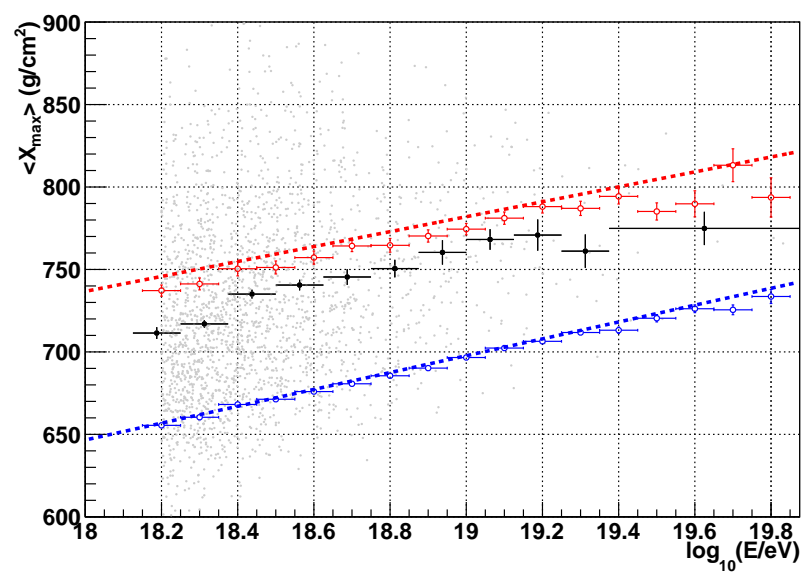

Figure 4: The mean $\mathrm{X}_{x \max }$ with the primary energy. The black points are TA hybrid 6 years hybrid data. The dotted lines show original mean $\mathrm{X}_{x \max }$ obtained from the Corsika output. The opened circles are predicttions obtained by the reconstructed MC data. The red and blue colors show QGSJET-II-03 proton and iron, respectively. 
within $21 \%$ energy scale uncertainty. The mass composition measurement performed by observing the depth of shower maximum $\left(\mathrm{X}_{\max }\right)$ is consistent with a light primary model.

\section{Acknowledgements}

The Telescope Array experiment is supported by the Japan Society for the Promotion of Science through Grants-inAid for Scientific Research on Specially Promoted Research (21000002) "Extreme Phenomena in the Universe Explored by Highest Energy Cosmic Rays" and for Scientific Research (19104006), and the Inter-University Research Program of the Institute for Cosmic Ray Research; by the U.S. National Science Foundation awards PHY-0307098, PHY- 0601915, PHY-0649681, PHY-0703893, PHY-0758342, PHY-0848320, PHY-1069280, PHY-1069286, PHY1404495 and PHY-1404502; by the National Research Foundation of Korea (2007-0093860, R3210130, 2012R1A1A2008381, 2013004883); by the Russian Academy of Sciences, RFBR grants 11-02-01528a and 13-02-01311a (INR), IISN project No. 4.4502.13, and Belgian Science Policy under IUAP VII/37 (ULB). The foundations of Dr. Ezekiel R. and Edna Wattis Dumke, Willard L. Eccles, and George S. and Dolores DorÂt'e Eccles all helped with generous donations. The State of Utah supported the project through its Economic Development Board, and the University of Utah through the Office of the Vice President for Research. The experimental site became available through the cooperation of the Utah School and Institutional Trust Lands Administration (SITLA), U.S. Bureau of Land Management, and the U.S. Air Force. We also wish to thank the people and the officials of Millard County, Utah for their steadfast and warm support. We gratefully acknowledge the contributions from the technical staffs of our home institutions. An allocation of computer time from the Center for High Performance Computing at the University of Utah is gratefully acknowledged.

\section{References}

[1] T. Abu-Zayyad et al. , Astropart. Phys. 61 (2015) 93-101.

[2] F. Kakimoto et al. , Nucl. Instrum. Meth. A 372 (1996) 527-533.

[3] R.U. Abassi et al. , Astropart. Phys. 29 (2008) 77-86.

[4] T. Tomida et al. , Nucl. Instrum. Meth. A 654 (2011) 653-660.

[5] H. Tokuno et al. , Nucl. Instrum. Meth. A 601 (2009) 364-371.

[6] B.T. Stokes et al. , Astropart. Phys. 35 (2012) 759-766. 